1 Fundação Oswaldo Cruz (Fiocruz), Escola Nacional de Saúde Pública Sergio Arouca (Ensp), Centro de Estudos da Saúde do Trabalhador e Ecologia Humana (Cesteh) - Rio de Janeiro (RJ), Brasil. leandrovbcarvalho@yahoo. combr

2 Fundação Oswaldo Cruz (Fiocruz), Escola Nacional de Saúde Pública Sergio Arouca (Ensp), Centro de Estudos da Saúde do Trabalhador e Ecologia Humana (Cesteh) - Rio de Janeiro (RJ), Brasil. costa.isabele@gmail.com

${ }^{3}$ Fundação Oswaldo Cruz (Fiocruz), Escola Nacional de Saúde Pública Sergio Arouca (ENSP), Centro de Estudos da Saúde do Trabalhador e Ecologia Humana (Cesteh) - Rio de Janeiro (RJ), Brasil. mattos@ensp.fiocruz.br

4 Fundação Oswaldo Cruz (Fiocruz), Escola Naciona de Saúde Pública Sergio Arouca (Ensp), Centro de Estudos da Saúde do Trabalhador e Ecologia Humana (Cesteh) - Rio de Janeiro (RJ), Brasil.

arilarentis@yahoo.com.br

\section{Exposição ocupacional a substâncias químicas, fatores socioeconômicos e Saúde do Trabalhador: uma visão integrada}

\author{
Occupational exposure to chemicals, socioeconomic factors and \\ Occupational Health: an integrated vision
}

Leandro Vargas Barreto de Carvalho', Isabele Campos Costa-Amaral2 ${ }^{\mathbf{2}}$ Rita de Cássia Oliveira da Costa Mattos ${ }^{3}$, Ariane Leites Larentis $\mathbf{4}$

RESUMO A relação entre exposições ocupacionais, compostos químicos e doenças é conhecida há muito tempo e está no cerne do campo saúde-trabalho-ambiente. A pobreza presente na vida de numerosos trabalhadores brasileiros cria um cenário de vulnerabilidade social, gerando riscos à saúde. Este estudo tem por objetivo discutir a importância dos fatores socioeconômicos na avaliação da exposição ocupacional e a relação com o processo de saúde/doença dos trabalhadores, por meio de uma revisão exploratória da literatura. Diversos fatores estão ligados à forma como o trabalhador se relaciona com a exposição, como as condições de vida, moradia, acesso a serviços de saúde e educação, nível salarial e escolar.

PALAVRAS-CHAVE Exposição ocupacional. Compostos químicos. Fatores socioeconômicos. Saúde do trabalhador.

ABSTRACT The relationship between occupational exposures, chemical compounds and diseases has been known for a long time and is at the heart of the health-work-environment field. The condition of poverty in the lives of many Brazilian workers creates a scenario of social vulnerability, generating risks to health. This study aims to discuss the importance of socioeconomic factors in the evaluation of occupational exposure and the relationship with workers' health/ illness process, through an exploratory review of the literature. Several factors are related to how the worker relates to exposure, such as living conditions, housing, access to health and education services, salary levels and schooling.

KEYWORDS Occupational exposure. Chemical compounds. Socioeconomic factors. Occupational health. 


\section{Introdução}

A relação entre as exposições ocupacionais e o aparecimento de doenças já é conhecida há muito tempo. Em 1556, Georg Bauer divulgou a primeira obra descrevendo a associação entre o trabalho de mineração e a manifestação de doença respiratória, chamada de re metallica. Já Bernardino Ramazzini, considerado o pai da medicina do trabalho, publicou na Itália, em 1700, o livro 'De morbis artificum diatriba' (as doenças dos trabalhadores), no qual descreve 54 doenças relacionadas com o trabalho e introduz na anamnese clínica a pergunta: 'qual é a sua ocupação?' (BAGATIN; KITAMURA, 2005). Em 1845, Friedrich Engels também denunciou as condições dos trabalhadores em 'A situação da classe trabalhadora na Inglaterra' (ENGELS, 1998). Desde então, diversos estudos foram realizados, evidenciando essa relação causal tão expressiva, e ainda hoje pouco valorizada. Essas obras começaram a discutir como a saúde (e as doenças) de um indivíduo, ou de uma classe, podem estar diretamente relacionadas com a sua atividade profissional, e, assim, plantaram a semente do que hoje se entende, de forma mais ampla e complexa, como Saúde do Trabalhador (ST). Porém, mesmo após tanto tempo de existência, a ST ainda precisa de mais atenção, para que, de fato, essas relações sejam compreendidas.

Neste artigo, pretende-se discutir tais relações com foco nas exposições ocupacionais a substâncias químicas. Esta questão se configura como um problema de saúde coletiva, pois está diretamente ligada com questões envolvendo a temática saúde-trabalho-ambiente. Uma vez que a maioria dos processos produtivos gera exposição a algum tipo de substância química, seja como meio reacional ou produto de reação, os trabalhadores estão direta ou indiretamente expostos a líquidos, gases, vapores ou aerodispersoides, durante parte ou toda jornada de trabalho. Devido à pluralidade de vias e formas de exposição, o indivíduo sempre estará em risco em um ambiente de trabalho, fato este com influência em seu estado de saúde, podendo gerar um quadro de adoecimento (AMORIM, 2003; OGA; CAMARGO; BATISTUZZO, 2008).

Embora as populações possam ser afetadas por fontes naturais (exposições ambientais), tais como erupções vulcânicas ou depósitos naturais de substâncias de elevada toxicidade, em geral, as poluições ambientais antropogênicas têm como principal origem os processos produtivos (TAMBELLINI; CÂMARA, 1998).

A Organização Internacional do Trabalho (OIT) estimou, em 2004, em 35 milhões de casos anuais de doenças relacionadas com o trabalho por exposição a substâncias químicas com a ocorrência de 439 mil mortes, incluindo, entre outras causas relacionadas, 36 mil óbitos por pneumoconioses, 35,5 mil óbitos por doenças respiratórias crônicas, 30,7 mil óbitos por doenças cardiovasculares e 315 mil óbitos por câncer. A Organização Mundial da Saúde (OMS), em 2002, estimou que esses cânceres provoquem uma perda anual de 1,4 milhão de anos de vida saudável, e que as intoxicações agudas por produtos químicos sejam responsáveis por outros 7,5 milhões de anos de vida saudável perdidos pela população mundial. Isso mostra que há muito o que se discutir a respeito da origem, do controle e da prevenção desses problemas, que não são recentes, embora cada vez mais novas substâncias químicas cheguem ao mercado (KATO; GARCIA; WUNSCH FILHO, 2007).

Apenas para que se tenha uma ideia do cenário brasileiro, em termos de exposições agudas, em 2012, o Sistema Nacional de Informações Tóxico-Farmacológicas (Sinitox) registrou 99.035 casos de intoxicação humana por agentes tóxicos, sendo que $5.989(6,0 \%)$ casos foram em ambiente ocupacional (BRASIL, 2012). Destes casos, 883 foram causados por produtos químicos industriais, e 1.172 foram causados por agrotóxicos. Cabe lembrar que esses sistemas de notificação normalmente são subdimensionados, pois acidentes de trabalho muitas vezes não são 
registrados, não refletindo o real tamanho do problema, o que leva a crer que a situação de risco aqui discutida deva ser maior que o exposto. Contudo, o sistema registra intoxicações humanas agudas, comumente associadas a acidentes, e, na realidade ocupacional, as exposições crônicas têm maior frequência em relação aos efeitos na saúde, porém não existe a mesma notificação. Os dados do Sinitox (2012) indicam que a intoxicação humana por substâncias químicas em ambientes ocupacionais é relevante e merece atenção nas discussões sobre saúde pública, uma vez que estes acidentes e seus desdobramentos irão fazer parte da rotina de atendimento do Sistema Único de Saúde (SUS). Assim, tentar entender as relações de causalidade implícitas nesses casos pode gerar ações de prevenção mais eficazes. Inclusive, a ST é uma atribuição, entre diversas outras, do SUS (art. $6^{\circ}$ ), contemplada na Lei Orgânica de Saúde (BRASIL, 1990), e em geral, preterida por envolver questionamento direto das empresas e implicar enfrentamentos com o capital.

O trabalho, enquanto fator imprescindível ao desenvolvimento técnico, econômico e social, tem representado um papel de importância ao longo de toda a história da humanidade (UVA; FARIA, 2000). Ele representa meio de subsistência, status, formação, identidade e lugar social. Ele ainda pode ter mais significações, dependendo da cultura e contexto social. Dessa forma, considerar a questão dos determinantes sociais da saúde e sua influência nas exposições ocupacionais traz uma abordagem mais completa de como o trabalhador interage com o ambiente insalubre e os riscos da exposição. De forma bem generalizada, Determinantes Sociais de Saúde (DSS) expressam o conceito de que as condições de vida e trabalho dos indivíduos e de grupos da população estão relacionadas com sua situação de saúde. Para a Comissão Nacional sobre os Determinantes Sociais da Saúde (CNDSS), os DSS são os fatores sociais, econômicos, culturais, étnicos/ raciais, psicológicos e comportamentais que influenciam a ocorrência de problemas de saúde e seus fatores de risco na população. A Comissão sobre DSS da OMS adota a definição na qual os DSS são as condições sociais em que as pessoas vivem e trabalham (BUSS; PELLEGRINI, 2007). Assim, fica mais evidente como o trabalho de um indivíduo afeta sua condição de saúde e como está diretamente ligado às suas condições de vida, ou seja, forma-se uma rede de fatores indissociáveis.

A Lei Orgânica de Saúde (BRASIL, 1990, P. 1) já ditava como norte que:

A saúde tem como fatores determinantes e condicionantes, entre outros, a alimentação, a moradia, o saneamento básico, o meio ambiente, o trabalho, a renda, a educação, o transporte, o lazer e o acesso aos bens e serviços essenciais; os níveis de saúde da população expressam a organização social e econômica do país.

Isso mostra que esse debate apenas relembra o papel importante da realidade socioeconômica quando se pensa em avaliação de saúde. Não esquecendo que tal fato é hoje reforçado pela Política Nacional de Saúde do Trabalhador e Trabalhadora (Portaria $\mathrm{n}^{\mathrm{o}}$ 1.823, de 23 de agosto de 2012, do Ministério da Saúde), em que fica mais clara ainda a importância da transversalidade das ações de ST e o trabalho como um dos determinantes do processo saúde-doença.

Ainda de acordo com Buss e Pellegrini (2007), o principal desafio dos estudos sobre as relações entre determinantes sociais e saúde consiste em estabelecer uma hierarquia de determinações entre os fatores mais gerais de natureza social, econômica, política e as mediações por meio das quais esses fatores incidem sobre a situação de saúde de grupos e pessoas, já que a relação de determinação não é uma simples relação direta de causa-efeito.

No modelo de Dahlgren e Whitehead, os DSS estão dispostos em diferentes camadas, 
desde uma camada mais próxima dos determinantes individuais, de menor peso, até uma camada distal, em que se situam os macrodeterminantes. $\mathrm{O}$ modelo não pretende explicar com detalhes as relações e mediações entre os diversos níveis e a gênese das iniquidades. Os fatores relacionados com as condições de vida e de trabalho, disponibilidade de alimentos e acesso a ambientes e serviços essenciais, como saúde e educação, indicam que as pessoas em desvantagem social correm um risco diferenciado, criado por condições habitacionais mais humildes, exposição a condições mais perigosas ou estressantes de trabalho e acesso menor aos serviçOS (BUSS; PELLEGRINI, 2007).

Diante do contexto, no qual o processo de adoecimento de um trabalhador exposto a um ambiente insalubre pode estar ligado a uma realidade social, inerente ao seu status socioeconômico, desenha-se o conceito de vulnerabilidade social, aqui representado como uma série de fatores que propiciam uma somatização e que implicam em diferenças nos tipos e na extensão dos impactos dos riscos sofridos, tais como, estado nutricional, escolaridade, possibilidades de acesso à informação, grau de qualidade das políticas públicas, moradia, entre outros (RIGOTTO; AUGUSTO, 2007).

Assim, os riscos são desigualmente distribuídos pela classe trabalhadora e influenciam em seu estado de saúde e como ou porque adoecem. Portanto, a relação entre as exposições ocupacionais e a realidade socioeconômica dos trabalhadores se configura como uma relação de potencialização de riscos, em que a pobreza e seus desdobramentos interagem com os riscos da exposição, uma vez que a interação desses fatores não tem como resultado um efeito apenas aditivo, mas sim uma complexificação de interações que levam a um resultado mais perigoso.

A abordagem das relações de trabalho e saúde-doença parte da ideia do corpo como máquina, exposto a agentes ou fatores de risco. Assim, as consequências do trabalho para a saúde são resultado da interação do corpo com agentes (físicos, químicos, biológicos, mecânicos), existentes no meio (ambiente) de trabalho, que mantêm uma relação de externalidade aos trabalhadores (LACAZ, 2007), potencializado no sistema capitalista que o trabalhador não detém o controle dos meios de produção, do ritmo de trabalho nem do produto final (MARX, 1988).

A salubridade dos ambientes de trabalho constitui um elemento relevante em matéria de saúde e bem-estar dos trabalhadores e não deve ser entendida como um mero problema de gestão de riscos profissionais confinado ao(s) local(is) de trabalho. Deve, sim, e de modo bem menos redutor, ser equacionada na perspectiva globalizante e integradora que caracteriza as ações de promoção da saúde (UVA; FARIA, 2000). Um processo de trabalho sem riscos ou a completa salubridade de um ambiente de trabalho é quase impossível, já que concentrações ou risco zero são abstrações (PIVETTA ET AL., 2001), considerando as atuais realidades ocupacionais e ambientais dos processos produtivos do capitalismo, ainda mais no Brasil, que abarca processos altamente poluentes. Do ponto de vista do campo da ST, a adequação de processos e ambientes faz parte da luta dos trabalhadores por melhores condições de trabalho e de vida, buscando processos de trabalho mais saudáveis para eles (LACAZ, 2007).

Nesse contexto do campo, para entender como que a ST não é apenas uma medida baseada em monitoramentos, que não são apenas números acima ou abaixo de limites pré-estabelecidos, e sim uma relação entre diversos fatores condicionantes, é importante definir e compreender o que é o monitoramento no contexto da avaliação da exposição ocupacional em ambientes de trabalho. A detecção precoce de uma exposição perigosa pode diminuir significativamente a ocorrência de efeitos adversos na saúde. O monitoramento da exposição é um 
procedimento que consiste em uma rotina de avaliação e interpretação de parâmetros biológicos e/ou ambientais, com a finalidade de detectar os possíveis riscos à saúde. A exposição pode ser avaliada pela medida da concentração do agente químico em matrizes ambientais - Monitoramento Ambiental (MA) - ou por meio da medida de parâmetros biológicos - Monitoramento Biológico (MB) - denominados indicadores biológicos ou biomarcadores. As informações provenientes do monitoramento da exposição possibilitam a implantação de medidas de prevenção e controle apropriadas para minimizar os efeitos sobre a saúde dos trabalhadores (AMORIM, 2003).

A estratégia de prevenção dos riscos ocupacionais engloba, sistematicamente, a abordagem simultânea da exposição ambiental (MA) e dos efeitos e/ou respostas (MB) individuais por ela provocados (OGA; CAMARGO; BATISTUZZO, 2008). Tal abordagem, para ser completa e fiel às relações de saúde/trabalho/ambiente - doença, deve considerar o trabalho como um todo, ou seja, respeitando as relações humanas do ambiente onde ocorre o trabalho e verificando como as condições socioeconômicas do trabalhador influenciarão na exposição.

A importância de como as condições sociais em que as pessoas vivem e trabalham influenciam sua saúde é discutida desde o final do século XIX, tendo tido várias abordagens diferentes no decorrer do tempo, porém sempre focada nos fatores e mecanismos por meio dos quais as condições sociais afetam a saúde e que potencialmente podem ser alterados pela luta dos trabalhadores pela saúde, que inclui conhecer o processo de trabalho e os riscos a que estão expostos (BUSS; PELLEGRINI, 2007; MARX, 1988).

Dessa forma, explicar como as diferentes abordagens (qualitativa e quantitativa) usadas para avaliação da ST podem estar intrinsecamente ligadas, ampliando a visão de avaliação da exposição, faz com que o processo de adoecimento seja entendido como algo mais complexo, não sendo somente uma série de números que determinam se o indivíduo está acima ou abaixo de faixas de risco, mas sim se o trabalhador em questão tem sua saúde prejudicada e por que isso está ocorrendo em sua vida, não somente em seu ambiente de trabalho. Assim, políticas de saúde, por parte dos órgãos competentes, tratariam o problema de forma mais completa, já que contemplariam uma gama de ações mais eficazes.

Este estudo surge da experiência dos trabalhos desenvolvidos, a partir de uma perspectiva de atendimento integral, multiprofissional e interdisciplinar, em um Ambulatório de Saúde do Trabalhador e em um Laboratório de Toxicologia, onde diversos trabalhadores, dos mais variados tipos de atividades profissionais, demonstram em seus históricos ocupacionais que não somente a definição de valores de exposição é suficiente para melhorar a condição de trabalho, mas sim uma maior compreensão de como e por que esses trabalhadores adoecem. Dessa forma, o objetivo deste trabalho foi discutir a relação existente entre as exposições ocupacionais a substâncias químicas, fatores socioeconômicos e o processo de adoecimento a partir destas experiências.

\section{Material e métodos}

Este foi um estudo de revisão exploratória da literatura, realizado até 2010 , por meio de consulta à Biblioteca Virtual em Saúde (BVS) (http://bvsalud.org/), utilizando as bases de dados Lilacs (Literatura LatinoAmericana e do Caribe em Ciências da Saúde) e Medline (Sistema Online de Busca e Análise de Literatura Médica). Os seguintes descritores (validades previamente verificadas no próprio portal da BVS, http://decs.bvs.br) foram usados para realizar uma busca sistematizada: occupational exposure AND socioeconomic factors AND chemicals. Os artigos relacionados 
pela BVS foram avaliados e selecionados para revisão. A busca de artigos completos foi feita no Portal de Periódicos Capes (Coordenação de Aperfeiçoamento de Pessoal de Nível Superior) (http://www. periodicos.capes.gov.br).

Foram utilizados como critério de inclusão artigos disponíveis na íntegra, nos idiomas português, inglês ou espanhol e que continham os descritores da pesquisa nos campos 'título' ou 'resumo' ou 'assunto'; e como critério de exclusão, resumos de artigos, artigos em outros idiomas e artigos não disponíveis eletronicamente.

A análise do crescimento dos artigos encontrados na busca por década foi feita empregando teste $\mathrm{t}$-Student com nível de significância de $95 \%$.

\section{Resultados e discussão}

O resultado da revisão gerou 55 referências, sendo que apenas 17 (31\%) delas estavam disponíveis eletronicamente e dentro dos critérios de inclusão (a maior parte delas após o ano 2000).

O gráfico 1 apresenta o número total de artigos encontrados na busca (55), distribuídos nas últimas quatro décadas (1970-2010), mostrando um crescimento linear e significativo ( $\mathrm{r} 2=0,9552$ e p-valor $=0,023)$ das publicações sobre o tema, ao longo do tempo. Os resultados indicam que a questão social referente à saúde dos trabalhadores é uma visão mais recentemente discutida; e no que tange ao tema exposição ocupacional a substâncias químicas, ainda carece de mais estudos.

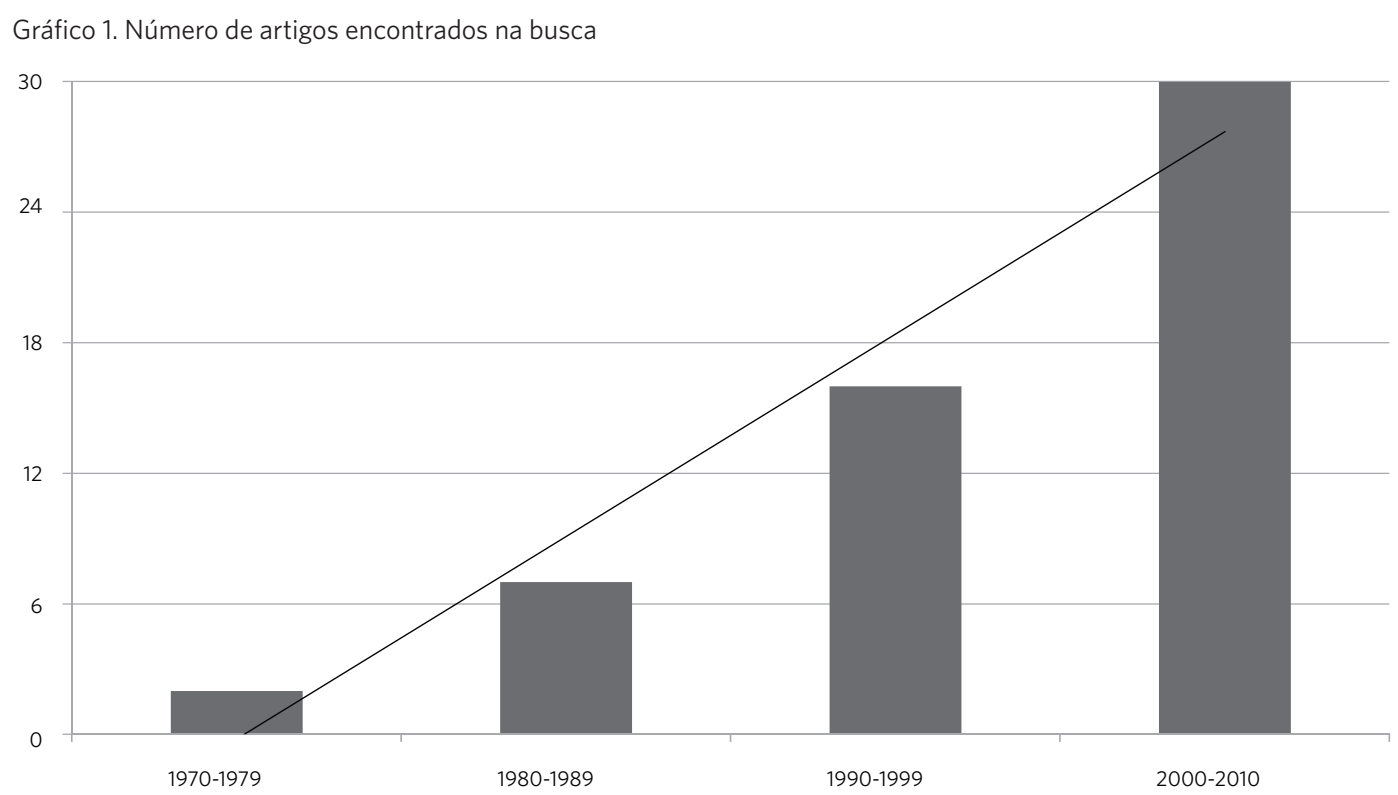

Fonte: Elaboração própria.

Esta revisão buscou verificar as perspectivas que a literatura apresentava sobre os temas exposições ocupacionais a substâncias químicas, fatores socioeconômicos e ST. Dentre os 17 artigos disponíveis para a revisão, após análise crítica do conteúdo, foram selecionados os que realmente abordavam as ideias relacionadas com os temas. Posteriormente, foram discutidas ideias que abrangessem todos estes pontos de vista estudados, salientando a importância de cada um isoladamente e, depois, mostrando a vantagem de uma visão integradora.

Uma parte das referências estudadas 
$(\mathrm{n}=6)$ indica que determinadas classes profissionais ou atividades desenvolvidas (processo de trabalho) com substâncias químicas específicas geram um maior risco para o desenvolvimento de algumas doenças, porém, os artigos não verificam a influência que os fatores socioeconômicos podem exercer, ou seja, estes não são considerados fatores determinantes, ou condicionantes de doença dentro do estudo, apenas características (sociodemográficas) descritivas importantes da população a ser estudada (FATMA; JAIN; RAHMAN, 1991; FÉRRIS I TORTAJADA ET AL., 2005; Ji; HEMMINKI, 2006; KRISHNADASAN ET AL., 2007; MAO ET AL., 2000; PANT, 2008).

No artigo de Fatma, Jain e Rahman (1991), foi verificada a frequência de danos genéticos em 22 trabalhadores de fábrica de cimento-amianto. O contexto social foi apenas considerado para o grupo controle de 12 pessoas, ajustados em idade similar, sexo, hábitos e status socioeconômico, não demonstrando que fatores são considerados para definir o status.

No artigo de Mao et al. (2000), foi feito um estudo de caso-controle, entre 1994 e 1997, com 1.469 pessoas envolvidas (casos) e um grupo controle de 5.073 pessoas, para verificar a formação de linfoma Não Hodgkin entre trabalhadores canadenses e sua associação com a exposição a substâncias químicas. Foi calculada a razão de chances (odds ratio) para renda familiar e tempo de estudo, entre casos e controles, que ficou dentro da normalidade, porém, estes dados não foram cruzados com as substâncias químicas.

$\mathrm{Na}$ revisão bibliográfica de Férris i Tortajada et al. (2005), foram elencados os fatores de risco para o desenvolvimento de neuroblastoma. Os autores concluíram que os seguintes fatores incrementam o risco, com maior ou menor evidência científica: genéticos, geográficos, étnicos, socioeconômicos, infecciosos, físicos, exposições ocupacionais dos pais, gestacional, materna e perinatal.

Já no estudo de Ji e Hemminki (2006), foram considerados fatores socioeconômicos e ocupacionais para verificar a associação entre eles e doenças linfoproliferativas; porém, basicamente o que os autores apresentaram em seu artigo foi uma categorização de ocupações, de acordo a Classificação Nórdica de Ocupações. O estudo concluiu que esses fatores têm um menor efeito sobre o risco de doença do que a exposição a solventes orgânicos, algo paradoxal, pois, no caso, a exposição é oriunda do processo de trabalho, mostrando a limitação em não empregar os conceitos do campo da ST na formulação do estudo.

No trabalho de Krishnadasan et al. (2007), foi realizado um estudo caso-controle retrospectivo (entre 1950 e 1992) para verificar a incidência de câncer de próstata entre 362 casos (trabalhadores de uma usina americana de energia nuclear e motor de foguetes) e 1.805 controles. Foi considerado como fator socioeconômico o que os autores denominaram como fator ocupacional de confundimento: o vínculo trabalhista (profissional e não profissional assalariado e horas/trabalho), que foi ajustado nas categorias descritas e, posteriormente, feita a verificação da associação entre a doença e as substâncias químicas. Os autores indicaram que altos níveis de exposição a tricloroetilieno estavam associados com câncer de próstata entre os trabalhadores da população estudada.

No estudo de Pant (2008), populações masculinas urbanas e rurais foram avaliadas quanto à qualidade do sêmen devido à exposição a ftalatos (oriundo de diferentes atividades industrial). Os fatores socioeconômicos (como nível educacional e status social, de acordo denominação dos autores no artigo) foram considerados no questionário de características demográficas.

Outros trabalhos $(n=3)$ associaram a exposição ocupacional a substâncias químicas e os fatores socioeconômicos com maior risco à saúde, entre eles, Suadicani, Hein e Gyntelberg (1995), Niedhammer et al. (2008) e Porta et al. (2008).

No artigo de Suadicani, Hein e Gyntelberg (1995), foi feito um estudo de coorte com 2.974 homens, no período de 1985 a 1991, para 
examinar se as condições físicas e químicas de trabalho explicavam a associação de classe social com doença isquêmica cardíaca (DIC). A classe social, classificada em 5 categorias, baseadas em nível educacional e perfil de trabalho, foi considerada como fator de confundimento. Os autores concluíram que diferenças sociais na exposição aos fumos de solda e solventes orgânicos são responsáveis por aumento do risco de DIC em classes sociais mais baixas.

No estudo de Porta et al. (2008), foram verificadas as associações entre fatores sociais e concentrações séricas de agentes químicos ambientais no desenvolvimento de câncer pancreático. Como os agentes químicos descritos foram compostos organoclorados (agrotóxicos), a exposição a eles é dada no dia a dia do trabalhador rural. Foi realizado um estudo prospectivo com 135 pacientes de um hospital, e a classe social foi categorizada em 5 grupos, baseada em perfil de trabalho. Nível educacional foi considerado na entrevista para responder ao questionário. Os autores concluíram que concentrações de organoclorados foram maiores em classes sociais mais baixas e que a incidência de câncer de pâncreas não pode ser avaliada independente da classe social nesse tipo de caso. Portanto, é necessária a integração de evidências que contribuam para entender a etiologia da doença, do ponto de vista social e da exposição ocupacional.

Um dos trabalhos mais importantes desta revisão, o de Niedhammer et al. (2008), apresenta uma discussão qualitativa (valendo-se de resultados quantitativos), algo não presente nas outras referências. Os autores debatem que as desigualdades sociais em saúde têm sido demonstradas, mas a compreensão destas desigualdades permanece obscura, e acrescenta que o trabalho e os respectivos fatores ocupacionais podem contribuir para essas desigualdades. O estudo francês contou com a participação de 14.241 homens e de 10.245 mulheres trabalhadoras, provenientes de um inquérito nacional da população. Os fatores incluíram características do trabalho, a exposição ocupacional aos riscos físicos, ergonômicos, químicos, biológicos e psicossociais e o ambiente de trabalho. Como resultado, fortes gradientes sociais, termo utilizado pelos autores, foram verificados em todos os fatores de trabalho analisados. Gradientes sociais também foram observados para os desfechos de saúde estudados, sendo os trabalhadores manuais mais propensos a relatar problemas de saúde, faltas por doença e acidentes de trabalho. $\mathrm{O}$ estudo sugere que a prevenção dos fatores de risco ocupacionais seria útil, não só para melhorar a saúde no trabalho, mas também para reduzir as desigualdades sociais em saúde.

$O$ restante dos artigos $(n=8)$, representando a metade dos disponíveis para revisão, apesar de possuírem os descritores da pesquisa nos campos 'título ou resumo ou assunto', não relacionaram as ideias dos temas entre si, portanto não foram abordados nesta discussão.

Goldberg (2002) traz um elemento importante para o debate 'exposição ocupacional e fatores socioeconômicos'. O autor discute que os 'modelos' para análises que levam em conta 'fatores' sociais como parâmetros em conjunto com os biológicos para explicar um fenômeno de saúde são simplistas, empiristas, reduzindo-os a 'fatores de risco' tratados como simétricos, de igual influência e 'habitualmente estudados ou isoladamente, ou por associação de um número bem reduzido de riscos', sem verificar suas inter-relações, o que pode explicar o fracasso de um grande número deles. $\mathrm{O}$ autor mostra que os estudos que consideram fatores sociais são uma minoria, o que foi corroborado por este trabalho, e critica a utilização da categoria 'fatores de risco' para o estudo da relação entre a saúde e os aspectos socioeconômicos. Ele ainda complementa que, a priori, não é muito satisfatório adotar um modelo linear no qual todas as variáveis são colocadas em um mesmo plano, ignorando suas inter-relações. 


\section{A realidade brasileira}

Segundo Rigotto e Augusto (2007, p. S481), "o Brasil não é um país pobre, mas tem um número extremo de pessoas pobres". Apesar de estar entre as maiores economias do mundo, milhões de brasileiros vivem na pobreza, sendo um país com grandes desigualdades sociais e econômicas. Dados recentes (MEDEIROS; SOUZA; CASTRO, 2015) mostram que, enquanto $50 \%$ da população detém apenas $10 \%$ da renda, $90 \%$ da população detém aproximadamente $40 \%$ da renda total brasileira. Isso significa que $10 \%$ da população detém $60 \%$ da renda, e caso se avance no topo dos maiores rendimentos, $0,5 \%$ da população detém $20 \%$ da renda brasileira. Esse é o retrato da extrema desigualdade do País, que está estável no período analisado pelos autores (2006, 2009 e 2012).

Em um artigo publicado em outubro de 2010, no site do Programa das Nações Unidas para o Desenvolvimento (PNUD), o título já inicia um debate que contém elementos orientadores para este trabalho: 'emprego, sozinho, não reduz miséria' (PNUD, 2010), remuneração e condições salubres de trabalho influem diretamente nas condições de vida e saúde dos trabalhadores.

Quando essa discussão é focada na temática saúde-trabalho-ambiente, a situação fica mais complexa, pois se cria um cenário propício para vulnerabilidades sociais. Como o objeto deste trabalho envolve a ST, essa vulnerabilidade determina um risco maior que a parcela mais humilde dos trabalhadores tem de adoecer diante de situações e ambientes de trabalho insalubres. Essa parcela citada tem uma realidade de vida sofrida, normalmente pontuada por trabalhos sem qualificação, baixa remuneração, exposições ocupacionais perigosas, além de moradias sem infraestrutura básica e/ou em locais perigosos, carência nutricional, falta de acesso a serviços básicos de saúde e educação, ausência de políticas públicas sociais, escolaridade baixa (ou mesmo, analfabetismo), enfim, uma gama de complexos fatores que interagem entre si, e tornam o indivíduo vulnerável a diversos problemas. É importante pontuar que o conceito de vulnerabilidade aqui utilizado se refere a uma predisposição que o trabalhador pobre ou carente apresenta, ante uma exposição ocupacional ou ambiental, de sofrer com agravos a sua saúde, devido a suas condições de trabalho e de vida.

Como exemplo desse cenário, tem-se o estudo de Oliveira-Silva et al. (2001), no qual é discutido que a utilização de agrotóxicos no meio rural brasileiro tem trazido uma série de consequências tanto para o ambiente como para a ST rural. Em geral, essas consequências são condicionadas por fatores intrinsecamente relacionados, tais como o uso excessivo dessas substâncias, a alta toxicidade dos produtos, a falta de utilização de equipamentos de proteção e a precariedade dos mecanismos de vigilância. Esse quadro ainda é agravado pelo baixo nível socioeconômico e cultural da grande maioria desses trabalhadores. Os autores demonstraram baixo percentual de indivíduos que leem os rótulos das embalagens, o que pode ser explicado pelos níveis de escolaridade encontrados na comunidade. Também comentam que, mesmo dentro desse grupo, é de se esperar que os textos não sejam perfeitamente interpretados, tanto pelo nível de escolaridade quanto pelo teor técnico das informações contidas nos rótulos, o que cria uma série de barreiras à comunicação sobre o uso, os cuidados e os efeitos sobre a saúde e o ambiente.

Entre os diversos fatores envolvidos na discussão sobre exposições ocupacionais e fatores socioeconômicos, provavelmente o que mais influencia diretamente em uma exposição ocupacional, principalmente no caso de substâncias químicas, é o analfabetismo (OLIVEIRA-SILVA ET AL., 2001). Socialmente, o analfabetismo por si só já é um problema de grandes proporções: demonstra falhas na educação básica de um país, falta de políticas públicas de educação, cerceia a ação do indivíduo, limitando sua vida cotidiana e dificultando ou impedindo o acesso aos serviços, como os de saúde e o conhecimento 
e luta por seus direitos, e, mais profundamente, lhe retira uma dignidade de cidadão. Quando se relaciona esse fator com uma realidade de exposição em ambientes de trabalho, isso gera um efeito catastrófico. $\mathrm{O}$ trabalhador que não sabe ler, não consegue se informar do risco. A informação não é interpretada ou compreendida, portanto o risco não é assimilado.

Um estudo que corrobora essa hipótese é o de Heron (1997). Nesse artigo foi avaliado um programa de educação de trabalhadores da indústria química para a prevenção de dermatoses. Ao fim de 3 anos, o autor verifica que o número de novos casos da doença cai de 70 para 27 , na mesma população, e que, ainda, os casos de dermatose passaram a durar menos dias $(7,22$ para 4,67$)$, aumentando o índice de recuperação. Esse estudo demonstra a importância da educação do trabalhador como uma ferramenta de prevenção primária de doenças. Materiais para treinos, como vídeos e apresentação de pôsteres, podem ser efetivos para a indústria na prevenção e controle de dermatoses.

A educação dos trabalhadores em seus locais de trabalho, juntamente com a instrução e incentivo para o uso de equipamentos de proteção individual, e, é claro, a presença dos equipamentos de proteção coletiva, que são obrigação das empresas, minimiza os riscos, embora não exclua a exposição a substâncias químicas, principalmente naqueles processos produtivos mais perigosos, poluentes, ou que incluam produtos cancerígenos e perigosos. Estudos de avaliação da exposição ocupacional devem contemplar palestras instrutivas/educativas aos trabalhadores, acerca dos riscos relacionados com o ambiente e processo de trabalho e devem ser propositivos para a elaboração de políticas públicas dentro do contexto saúde-trabalho-ambiente.

Um ponto que também merece destaque neste debate é a anamnese ocupacional, pois ela pode revelar dados diretamente ligados ao adoecimento do trabalhador, tanto oriundos do ambiente de trabalho quanto da degradação do meio ambiente, poluição atmosférica, que, entre outros tópicos, enfocam a emissão de substâncias químicas sabidamente causadoras de agravos à saúde. Infelizmente, fatores adversos como a formação inadequada do profissional de saúde, de tempo, ou, simplesmente, por não considerar o ambiente ou o trabalho como fator deletério à saúde humana, a grande maioria das anamneses ou fichas médicas não contemplam dados mínimos de história ocupacional ou ambiental (BAGATIN; KITAMURA, 2005).

Rigotto e Augusto (2007, P. 5478) discutem que movimentos sociais de diferentes lugares do mundo questionam a noção de igualdade na exposição a riscos, pois "a enorme concentração de poder na apropriação dos recursos ambientais" e as dinâmicas sociais e políticas que, em sociedades desiguais,

destinam a maior carga dos danos ambientais do desenvolvimento às populações de baixa renda, aos grupos raciais discriminados, aos povos étnicos tradicionais, aos bairros operários, às populações marginalizadas e vulneráveis.

Ainda sob a perspectiva ambiental, vale lembrar que a contribuição dessa via é de fundamental importância para o entendimento da contaminação humana por substâncias químicas. Acredita-se que um maior número de pessoas esteja exposto por meio dessa via, em relação à via ocupacional, entretanto, o impacto resultante da contaminação ambiental é, em geral, consideravelmente menor que o impacto resultante da via ocupacional (MOREIRA ET AL., 2002).

Diante da realidade brasileira de agressão à ST e ambiental, as últimas décadas registraram algumas lutas dos trabalhadores e dos movimentos sociais pela saúde e melhores condições de trabalho e de vida no Brasil: trabalhadores urbanos que lutam pelo banimento do amianto e de poluentes orgânicos persistentes ou que criam novas formas de destinar os resíduos produzidos; lutas pela 
definição do benzenismo a partir da constatação de síndromes mielodisplásicas e leucemias nos processos produtivos com benzeno; moradores de regiões metropolitanas que buscam garantir políticas adequadas de saneamento ambiental ou de moradia; comunidades ribeirinhas que defendem as terras das grandes barragens hidrelétricas; povos da Amazônia que também tentam proteger a floresta dos madeireiros, garimpeiros e especuladores (RIGOTTO; AUGUSTO, 2007).

Como o trabalho é a forma de reprodução da força de trabalho no sistema capitalista, além de representar, de forma ideológica, a vida social do indivíduo - e sem trabalho, ele perde sua identidade perante a sociedade -; um trabalhador que esteja sujeito a condições insalubres, mesmo sabendo que isso pode gerar um risco para sua saúde e um possível adoecimento, continuará no emprego, aceitando todos os riscos, conscientemente ou não, pois ele depende do trabalho para sua sobrevivência e reprodução social. Sem escolha, a não ser vender sua força de trabalho no sistema capitalista, em palavras claras, o trabalhador prefere se expor a riscos do que passar fome.

\section{Uma perspectiva de avaliação integrada}

Quando se realiza uma avaliação da exposição, certamente os monitoramentos biológico e ambiental se apresentam como ferramentas indispensáveis de análise, porém, anteriormente a isso, o estudo da situação real (o trabalho realizado, e não somente o prescrito) deve considerar as condições de trabalho.

As formas como o trabalhador está exposto (monitoramento) e como ele convive com essa situação (realidade socioeconômica) devem ser interpretadas conjuntamente, com intenção de entender o processo de adoecimento de forma o mais integral possível (MOREIRA ET AL., 2002). Um estudo de avaliação da exposição deve englobar esses diferentes aspectos relacionados com a ST. A figura 1 propõe como um estudo desse tipo pode ser conduzido: a avaliação da exposição como ideia central e a integração da avaliação dos aspectos relacionados com o processo e ambiente de trabalho, da avaliação médico/ clínica e do perfil socioeconômico e monitoramentos biológico e ambiental, assim como a análise de percepção de riscos.

Figura 1. Abordagem integrada em um estudo de avaliação da exposição

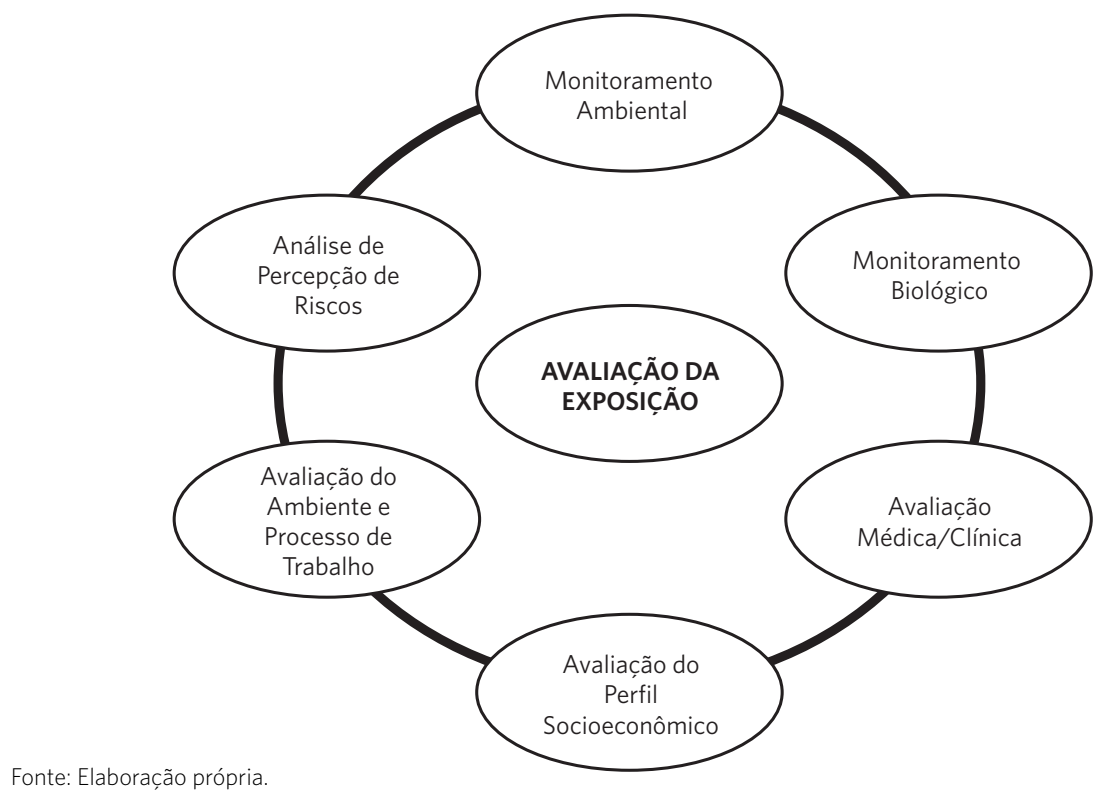


Esta perspectiva de avaliação integrada da exposição busca compreender o que está além do monitoramento, visando entender de que forma a exposição e o ambiente de trabalho influenciam a saúde de um trabalhador e como isso está ligado a sua realidade socioeconômica.

\section{Conclusões}

As exposições às substâncias químicas são uma parcela significativa dentro do campo das exposições ocupacionais, uma vez que essas substâncias estão presentes em vários ambientes e processos de trabalho. Compreender os determinantes sociais da saúde envolvidos nessas situações é importante para entender o processo de adoecimento, pois há uma potencialização de riscos relacionada com o perfil socioeconômico de um trabalhador e suas condições de trabalho, fato este intrincado em uma gama complexa de interações entre realidade social e exposições ocupacionais.

Os monitoramentos biológico e ambiental são ferramentas de análise imprescindíveis para a condução de um estudo de avaliação da exposição, porém, a forma como o trabalhador se relaciona com seu ambiente e processo de trabalho não são grandezas mensuráveis, e sim parâmetros que devem ser interpretados.

Durante o processo de avaliação da exposição, os fatores socioeconômicos devem ser considerados, não deixando de ser contemplados na anamnese ocupacional ou questionários, tornando-se parte da avaliação como um todo. Vários são os fatores socioeconômicos, indicados pela literatura, que influenciam a forma como o trabalhador se relaciona com sua exposição diária no ambiente de trabalho, tais como as condições de vida, moradia, o acesso a serviços de saúde e educação, nível de escolaridade, ligado à percepção do trabalhador em relação ao risco, nível salarial, assim como a luta dos trabalhadores por melhores condições de vida e trabalho, que lhes permite intervir direta e ativamente em sua condição de saúde.

Ações de vigilância devem intervir nos ambientes de trabalho, buscando uma melhora nos processos produtivos que torne menos insalubre a atividade do trabalhador, porém, sem uma ação política paralela, que contemple uma melhoria efetiva realidade social dos trabalhadores: buscando uma melhora na qualidade de vida, trabalhadores continuarão se expondo a ambientes perigosos, sem se preocupar com sua saúde.

A pobreza que está presente na vida de grande parte dos trabalhadores brasileiros cria um cenário de vulnerabilidade social, no qual o indivíduo fica mais propenso a adoecer ou sofrer um acidente de trabalho, pois ocorre uma naturalização ou aceitação do risco. Assim, enfrentar as questões políticas relacionadas com os processos de saúde/ doença e melhorar as condições de trabalho são ações fundamentais para a promoção da saúde dos trabalhadores.

\section{Colaboradores}

CARVAlHO, L. V. B. e LARENTIS, A. L. contribuíram substancialmente para a concepção, planejamento ou para a análise e a interpretação dos dados. COSTA-AMARAL, I. C. e MATTOS, R. C. O. C. contribuíram significativamente na elaboração do rascunho ou revisão crítica do conteúdo. 


\section{Referências}

AMORIM, L. C. A. Os biomarcadores e sua aplicação na avaliação da exposição aos agentes químicos ambientais. Revista Brasileira de Epidemiologia, São Paulo, v. 6, supl., p. 1-13, 2003. Disponível em: <http://www.scielosp.org/pdf/ rbepid/v6n2/09.pdf>. Acesso em: 8 mar. 2016.

BAGATIN, E.; KITAMURA, S. História Ocupacional. Jornal Brasileiro de e Pneumologia, Brasília, DF, v. 32, supl., p. 12-16, 2005.

BRASIL. Ministério da Saúde. Fundação Oswaldo Cruz (Fiocruz). Sistema Nacional de Informações Tóxicofarmacológicas (Sinitox). Dados de intoxicação. Casos registrados de intoxicação humana por agente tóxico e circunstância. Rio de Janeiro, 2012. Disponível em: <http:// sinitox.icict.fiocruz.br/sites/sinitox.icict.fiocruz.br/files// Tabela\%206_2012.pdf>. Acesso em: 26 jun. 2016.

Lei no 8.080, de 19 de setembro de 1990. Dispõe sobre as condições para a promoção, proteção e recuperação da saúde, a organização e o funcionamento dos serviços correspondentes e dá outras providências. Diário Oficial [da] União. Brasília, DF, 19 set. 1990. Disponível em: <http://www.planalto.gov.br/ccivil_03/ leis/L8080.htm>. Acesso em: 1 ago. 2016.

BUSS, P. M.; PELLEGRINI, A. F. A saúde e seus determinantes sociais. PHYSIS: Revista de Saúde Coletiva, Rio de Janeiro, v. 17, n. 1, p. 77-93, 2007. Disponível em: $<$ http://www.scielo.br/pdf/physis/v17nl/v17nla06. pdf>. Acesso em: 8 mar. 2016.

ENGELS, F. The Condition of the Working Class in England. Londres: [s. n.], 1998. Disponível em: <https:// www.marxists.org/archive/marx/works/download/ pdf/condition-working-class-england.pdf $>$. Acesso em: 1 ago. 2016.

FATMA, N.; JAIN, A. K.; RAHMAN, Q. Frequency of sister chromatid exchange and chromosomal aberrations in asbestos cement workers. British Journal of Industrial Medicine, v. 48, n. 2, p. 103-105, 1991. Disponível em: <https://www.ncbi.nlm.nih.gov/pmc/ articles/PMC1035328/>. Acesso em: 1 ago. 2016.
FÉRRIS i TORTAJADA, J. et al. Factores de riesgo para el neuroblastoma. Anales de Pediatría, Barcelona, v. 63, n. 1, p. 50-60, 2005. Disponível em: <http://www. analesdepediatria.org/es/factores-riesgo-el-neuroblastoma/articulo/13076768/>. Acesso em: 1 ago. 2016.

GOLDBERG, M. Este obscuro objeto da Epidemiologia. In: COSTA, D. N. (Org.). Epidemiologia: teoria e objeto. 3. ed. São Paulo: Hucitec, 2002. p. 87-136.

HERON, R. J. L. Worker education in the primary prevention of occupational dermatoses. Occupational Medicine, v. 47, n. 7, p. 407-410, 1997.

JI, J. I.; HEMMINKI, K. Socioeconomic/ occupational risk factors for lymphoproliferative diseases in Sweden. Annais of Epidemiology, Estados Unidos, v. 16, n. 5, p. 370-376, maio 2006. Disponível em: <http://www.sciencedirect.com/science/article/pii/ S1047279705003133>. Acesso em: 1 ago. 2016.

\section{KATO, M.; GARCIA, E. G.; WUNSCH FILHO, V.} Exposições a agentes químicos e a Saúde do Trabalhador. Revista Brasileira de Saúde Ocupacional, São Paulo, v. 32, n. 116, p. 6-10, 2007. Disponível em: <http:// www.scielo.br/scielo.php?script=sci_arttext\&pid =S0303-76572007000200002>. Acesso em: 1 ago. 2016

KRISHNADASAN, A. et al. Nested Case-Control Study of Occupational Chemical Exposures and Prostate Cancer in Aerospace and Radiation Workers, American Journal of Industrial Medicine, v. 50, n. 5, p. 383-390, 2007. Disponível em: <http://onlinelibrary.wiley.com/ doi/10.1002/ajim.20458/full\#references >. Acesso em: 1 ago. 2016.

LACAZ, F. A. C. O campo Saúde do Trabalhador: resgatando conhecimentos e práticas sobre as relações trabalho-saúde. Cadernos de Saúde Pública, Rio de Janeiro, v. 23, n. 4, p. 757-766, abr. 2007. Disponível em: <http://www.scielo.br/pdf/csp/v23n4/02.pdf >. Acesso em: 1 ago. 2016.

MAO, Y. et al. Non-Hodgkin's lymphoma and occupational exposure to chemicals in Canada. Annals of 
Oncology, Canadá, v. 11, supl., p. 69-73, 2000. Disponível em: <https://doi.org/10.1093/annonc/11.suppl_1.S69>. Acesso em: 1 ago. 2016.

MARX, K. O Capital: crítica da economia política. São Paulo: Nova Cultural, 1988. p. 21-27. v. 1.

MEDEIROS, M.; SOUZA, P. H. G. F; CASTRO, F. A. A estabilidade da desigualdade de renda no Brasil, 2006 a 2012: estimativa com dados do imposto de renda e pesquisas domiciliares. Ciência \&t Saúde Coletiva, Rio de Janeiro, v. 20, n. 4, p. 971-986, 2015. Disponível em: $<$ http://www.scielo.br/pdf/csc/v20n4/pt_1413-8123csc-20-04-00971.pdf>. Acesso em: 1 ago. 2016.

MOREIRA, J. C. et al. Avaliação Integrada do impacto do uso de agrotóxicos sobre a saúde humana em uma comunidade agrícola de nova Friburgo, RJ. Ciência Et Saúde Coletiva, Rio de Janeiro, v. 7, n. 2, p. 299-311, 2002. Disponível em: <http://www.scielo.br/pdf/csc/ v7n2/10249.pdf>. Acesso em: 1 ago. 2016.

NIEDHAMMER, I. et al. The contribution of occupational factors to social inequalities in health: Findings from the national French Sumer survey. Social Science Et Medicine, Nova Iorque, v. 67, p. 1870-1881, 2008.

OGA, S.; CAMARGO, M. M. A.; BATISTUZZO, J. A. O. (Ed.). Fundamentos de Toxicologia. 3. ed. São Paulo: Atheneu Editora, 2008.

OLIVEIRA-SILVA, J. J. et al. Influência de fatores socioeconômicos na contaminação por agrotóxicos, Brasil. Revista Saúde Pública, São Paulo, v. 35, n. 2, p. 130-135, 2001. Disponível em: $<$ http://www.scielo.br/scielo.php?pid=S0034$-89102001000200005 \&$ script $=$ sci_abstract\&tlng $=p t>$. Acesso em: 1 ago. 2016.

PANT, N. et al. Correlation of phthalate exposures with semen quality. Toxicology and Apllied Pharmacology, Nova Iorque, v. 231, n. 1, p. 112-116, 2008. Disponível em: <http://www.sciencedirect.com/science/article/pii/ S0041008X08001610>. Acesso em: 1 ago. 2016.

PIVETTA, F. et al. Monitoramento biológico: conceitos e aplicações em saúde pública. Cadernos de Saúde
Pública, Rio de Janeiro, v. 17, n. 3, p. 545-554, maio/jun. 2001. Disponível em: <http://www.scielo.br/pdf/csp/ v17n3/4638.pdf >. Acesso em: 1 ago. 2016.

PORTA, M. et al. Differences in serum concentrations of organochlorine compounds by occupational social class in pancreatic cancer. Environmental Research, v. 108, p. 370-379, 2008. Disponível em: <http://public-files.prbb.org/publicacions/a344ee60-c2e6-012b-a7a7000c293b26d5.pdf>. Acesso em: 1 ago. 2016.

\section{PROGRAMA DAS NAÇÕES UNIDAS PARA O} DESENVOLVIMENTO (PNUD). 2010. Disponível em: <http://www.pnud.org.br/Noticia.aspx?id=2411 $>$. Acesso em: 29 jun. 2016.

RIGOTTO, R. M.; AUGUSTO, L. G. S. Saúde e ambiente no Brasil: desenvolvimento, território e iniquidade social. Cadernos de Saúde Pública, Rio de Janeiro, v. 23, supl., p. S475-S501, 2007. Disponível em: <http://www.scielo.br/scielo.php?pid=S0102$-311 X 2007001600002 \&$ script=sci_abstract\&tlng=pt $>$. Acesso em: 1 ago. 2016.

SUADICANI, P.; HEIN, H. O.; GYNTELBERG, F. Do physical and chemical working conditions explain the association of social class with ischaemic heart disease? Atherosclerosis, Amsterdam, v. 113, n. 1, p. 63-69, 1995. Disponível em: <https://www.ncbi.nlm.nih.gov/ labs/articles/7755656/>. Acesso em: 1 ago. 2016.

TAMBELLINI, A. T.; CÂMARA, V. M. A temática saúde e ambiente no processo de desenvolvimento do campo da saúde coletiva: aspectos teóricos, conceituais e metodológicos. Ciência \& Saúde Coletiva, Rio de Janeiro, v. 3, n. 2, p. 47-59, 1998. Disponível em: <http:// www.scielo.br/scielo.php?script=sci_arttext\&pid $=$ S1413-81231998000200005>. Acesso em: 1 ago. 2016.

UVA, A. S.; FARIA, M. Exposição profissional a substâncias químicas: diagnóstico das situações de risco. Saúde Ocupacional, Rio de Janeiro, v. 18, n. 1, jan./jun. 2000.

Recebido para publicação em agosto de 2016

Versão final em novembro de 2016

Conflito de interesses: inexistente

Suporte financeiro: Fundação de Amparo à Pesquisa do Estado do Rio de Janeiro (Faperj) (№ do Processo: E-26/203.487/2015) 